S Research S Suare

\title{
Super heavy particle production in high energy heavy ion collision
}

Kurepin Alexey ( $\nabla$ kurepin@inr.ru )

Institute for Nuclear Research, Moscow https://orcid.org/0000-0002-1851-4136

\section{Research Article}

Keywords: high energy, heavy ion collisions, parton model

Posted Date: January 22nd, 2021

DOI: https://doi.org/10.21203/rs.3.rs-150643/v1

License: (a) (i) This work is licensed under a Creative Commons Attribution 4.0 International License. Read Full License 


\title{
Super heavy particle production in high energy heavy ion collision
}

\section{A.B.Kurepin}

Institute for Nuclear Research RAS, Moscow

\begin{abstract}
The estimate based on the parton model is made on the rate of production of Super Heavy Particle ( SHP ) in subthreshold collision of heavy ions at LHC. For the one-month run of lead-lead collision the yield of $16 \mathrm{TeV}$ particle is of the order of 70 per year.
\end{abstract}

\section{Introduction}

In the Grand Unification Theory it is assumed that the first massive particles which are responsible for the symmetry breaking up to the symmetry of the Standard Model at the distances larger than $10^{-29} \mathrm{~cm}$ have masses of the order of $10^{12} \mathrm{TeV}$. In the simple version of this Theory next masses arise only at distances about $10^{-16} \mathrm{~cm}$, where the particles with masses of the order of $10^{2} \mathrm{GeV}$ should be considered. They are connected to the breaking of SU(2) symmetry, so called Higgs scalar bosons. Therefore a rather artificial hypothesis was proposed on the existence of the " gauge dessert", i.e. no particles have masses between $0.2-10^{12} \mathrm{TeV}$. However, some other possibility for high mass production is predicted by Large Extra Dimension theories, where our $3+1$ dimensional world is considered as a " brane " in the higher dimensional space. Then the Planck mass can be brought down to a level of the order of one $\mathrm{TeV}^{1}$. Then Super Heavy Particles with masses of several TeV like a mini Black Holes could be produced if the extra dimension is equal to seven.

Additionally more complicated models has been proposed with multistep breaking of the symmetry at super high energy down to the symmetry of the Standard Model, where some new particles could be adopted with masses in the interval filling the "gauge dessert". One of the most interesting possibility provides the Theory based on new symmetry principle connecting the particles with different statistics, so called Supersymmetry Theory. New types of 
heavy quarks and leptons are introduced in this theory: $\mathrm{s}$ - quarks and $\mathrm{s}-$ leptons and corresponding supersymmetry partners. In that respect we can take into account new possibilities for producing the masses up to $10^{3} \mathrm{TeV}$ with the LHC with heavy ions as an opportunity to search for new super heavy supersymmetry particles. We can expect rather small coupling between these new types of physical objects and particles of the ordinary world. Therefore, one can look for super heavy particles with a large lifetime.

The unique possibility of relativistic heavy ion collisions is production with considerable probability of particles which are kinematically forbidden for nucleon-nucleon collisions with the same energy per nucleon. The essential increase in the "subthreshold" antiproton production cross section for nucleus-nucleus collision normalized per one nucleon in comparison to nucleon-nucleus collision was observed ${ }^{2-6}$. This increase can reach two order of magnitude and cannot be explained by Fermi motion in the incident nucleus ${ }^{7}$. (Supplementary Fig. 1 ). " Subthreshold" particle production could be due to multinucleon or multiquark correlation in heavy nuclei.

The total energy in the center of mass system for $\mathrm{Pb}-\mathrm{Pb}$ collisions at the LHC is approximately $1150 \mathrm{TeV}$. Therefore, the unique possibility could be considered to produce Super Heavy Particles ( SHP ) by nucleus - nucleus collisions with masses much larger than the center of mass energy in $\mathrm{p}-\mathrm{p}$ collisions.

\section{Parton model for particle production in heavy ion collision}

For the analysis of the subthreshold hadron production at intermediate energies the phenomenological parton model was proposed ${ }^{8}$. ( Supplementary Methods ). It was observed that the subthreshold and near threshold production cross section in proton - nucleus and nucleus collisions for pions, kaons and antiprotons could be expressed as a universal function of the scaling quark - parton parameters, such as the Bjorken scaling parameter $x$ for deep inelastic electron scattering but take into account the production of massive particles and now 
different in incident $(\mathrm{Z}$ ) and in target $(\mathrm{X}$ ) nuclei. Values $\mathrm{Z}$ and $\mathrm{X}$ larger than 1 arise to provide the production of particles at subthreshold energies for nucleon - nucleon process. As usually a physical interpretation of these scaling parameters in a quark-parton model means that $\mathrm{X} P_{b}$ correspond to the parton $4-$ momentum in a target nuclei, where $P_{b}$ is a $4-$ momentum of a nucleon in a target nucleus. $\mathrm{X}$ could take the values in an interval $0<\mathrm{X}<\mathrm{A}$, where $\mathrm{A}$ is the atomic number of a target. On the other hand the same parton model could be applied to the incident particle Z $\mathrm{Pa}$.

For the antiproton production the value of $\mathrm{X}$ for fixed $\mathrm{Z}$ could be obtained:

$$
\mathrm{X}=\frac{Z\left(P_{a} P_{d}\right)+Z m_{a} m_{n}+\frac{1}{2}\left(m_{n}^{2}-m_{d}^{2}\right)}{Z\left(P_{a} P_{b}\right)-Z m_{a} m_{b}-\left(P_{b} P_{d}\right)-m_{b} m_{n}}
$$

which is derived from the conservation of the 4- momentum in the collision. Here $P_{d}$ is the 4momentum of the antiproton, $m_{a} m_{b} m_{n}$-mass of the nucleon in the incident and in the target nuclei, $m_{d}$ - mass of the antiproton. Because of the exponential dependence of production cross section on the scaling parameters this equation matches the smallest values of $\mathrm{X}$ and $\mathrm{Z}$ parameters corresponding to the smallest energy in the center of mass of partons interaction.

For proton - nucleus and nucleus - nucleus interactions the experimental data over a wide energy interval for different incident and target nuclei could be approximated with the same scaling law. Those are the data for antiproton production with Ne and Ni nuclei with $1-2$ GeV/nucl. at GSI ${ }^{6}$, proton and carbon nuclei with $3.65 \mathrm{GeV} /$ nucl. at JINR ${ }^{2-3}$, (Supplementary Fig. 3 ) with deuteron beam at $\mathrm{KEK}^{5}$ and protons and a Si- beam at LBL for antiproton and $\mathrm{K}^{-}$ production ${ }^{4}$. (Supplementary Fig.2 ). The scaling in Fig.1 is observed with a Z- parameter equal 1 for proton beam, 1.3 for deuteron, 2 for carbon beam and 3 for $\mathrm{Si}, \mathrm{Ne}$, and Ni nuclei ${ }^{8}$. The production cross sections were reduced by $\left(\mathrm{A}_{1} \mathrm{~A}_{2}\right)^{0.43}$ according to A-dependence investigated in ${ }^{9}$ and due to the different absorption nuclear effects for pions, kaons and antiprotons. 
The considerable increase of the ratio of kaon to pion production rates in nucleus nucleus collision compared to the same ratio in deuteron - nucleus collision was also explained by the scaling dependence on the $\mathrm{Z}$ parameter for $\mathrm{Z}<1$, but with the parameter $\mathrm{X}=2$ for incident carbon nucleus and $\mathrm{X}=1.5$ for incident deuterons ${ }^{10}$.

The considerable increase of the production rates for kaons and antiprotons compared to pions in nucleus - nucleus collisions was interpreted as the indication of collective parton effect and was quantitatively reproduced by introduction of the scaling parameters larger than one. From Fig. 2 we see that the dependence of the Lorenz invariant inclusive cross section, or of the structure function, on the scaling parameter is close to the exponent. The "subthreshold" structure function in Fig. 2 could be approximated as:

$$
\left(\mathrm{A}_{1} \mathrm{~A}_{2}\right)^{-0.43} \cdot \mathrm{E}_{1} \frac{d^{3} \sigma}{d p^{3}}\left[\mathrm{mb} \mathrm{GeV}{ }^{-2} \mathrm{c}^{3} \mathrm{sr}^{-1}\right]=0.57 \exp (-\mathrm{X} / 0.158)
$$

where $\mathrm{A}_{1}$ and $\mathrm{A}_{2}$ are the mass numbers of colliding nuclei.

This curve can be used to describe the experimental data in the range of cross section $10^{-1}-10^{-5} \mathrm{mb}$ with the accuracy less than an order of magnitude for $\mathrm{X}=0.2-3$ for the data from approximately 2 to $6 \mathrm{GeV}$ per nucleon.

The enhancement of kaon and antiproton production is quantitatively reproduced by the introduction of a large $Z$ scaling parameter: $Z=1.3$ for deuterons, $Z=3$ for all heavy colliding nuclei. For incident protons $\mathrm{Z}$ is equal to one.

This scaling was observed for different quark flavors. Therefore one can expect that the model could be applied to some heavier unknown quarks and that these collective phenomena could be due to some general space - time properties of nucleus- nucleus interaction or to the universal structure function of a nucleus. 


\section{Estimate of production yield of Super Heavy Particle production}

From equation ( 2 ) we see that the cross section strongly depends on the parameter $\mathrm{X}$.

Therefore, to produce SHP with considerable probability one should look for the kinematics with smallest $\mathrm{X}$ and $\mathrm{Z}$ possible. To perform the estimation of SHP production cross section for LHC using the scaling dependence of structure function ( 2 ) for $M>>m_{n}$ we can determine $Z$ and $X$ from the relation on the threshold for SHP production:

$$
\sqrt{S}=2 \mathrm{E} \sqrt{X \cdot Z}=\mathrm{M}
$$

where $\mathrm{M}$ is the mass of SHP, $\mathrm{m}_{\mathrm{n}}$ is the mass of nucleon, $\mathrm{E}$ is the energy per nucleon of colliding nuclei.

To estimate the production rate of SHP with the mass M, the equation ( 2 ) could be used with $Z=3$ for nucleus - nucleus collision and $X$ to be determined from ( 3 ).

The absolute value of the momentum P of SHP is obtained from the equation

$$
\mathrm{X}=\frac{2 Z\left(E \cdot E_{1}-E \cdot P \cdot \cos \theta\right)-M^{2}}{4 Z \cdot E^{2}-2\left(E \cdot E_{1}+E \cdot P \cdot \cos \theta\right)}
$$

which is derived from the conservation of the 4- momentum in the collision. Here $E_{1}$ is the total energy of SHP, $\theta$ is the angle between SHP and $\mathrm{Z}$ parton.

Assuming that SHP are produced with small Pt in the narrow forward - backward cone we obtain for the masses M larger than $1 \mathrm{TeV}$, the velocities of SHP are of the order of beta= $0.1-0.9$.

Accepting that the multi quark correlations are the intrinsic property of nuclei and do not depend on the collision energy, we can try to use the "subthreshold" structure function ( 2 ) to estimate the production rate of SHP depending on their mass in $\mathrm{Pb}-\mathrm{Pb}$ collisions:

$$
\mathrm{E}_{1} \frac{d^{3} \sigma}{d p^{3}}\left[\mathrm{mb} \mathrm{GeV}^{-2} \mathrm{c}^{3} \mathrm{sr}^{-1}\right]=56 \exp (-\mathrm{X} / 0.158)
$$

For the obtained luminosity $\mathrm{L}=2 \cdot 10^{27} \mathrm{~cm}^{-2} \mathrm{sec}^{-1}$ for one month of the run and an opening angle of approximately $100 \mathrm{msr}$ for the forward detector we obtain an estimate of the 
SHP yield for the mass about $16 \mathrm{TeV}$ near the threshold on the order of 70 per year. For larger SHP masses the production cross section is greatly reduced due to the increasing parameter $\mathrm{X}$ in the exponential dependence.

The question remains about the possibility of applicability of the empirical scaling law, obtained at intermediate energies, to interactions at ultrahigh LHC energies. Unfortunately, it is impossible to measure the scattering and production of particles kinematically forbidden for nucleon - nucleon interaction at angles greater than 90 degrees on collider experimental facilities, that is, at $\mathrm{X}>1$.

Very promising possibilities for studying subthreshold phenomena are opened up in the case of experiments with a fixed target at the LHC ${ }^{11-13}$. The AFTER or ALICE-FT project provides for the placement of a solid or gaseous target in the beam halo when using all detectors of the ALICE facility. In this case, it is possible to measure the production of particles, in particular antiprotons, at large parameters $\mathrm{X}^{13}$ even at $\mathrm{X}>1$, which will make it possible to check the existence of the scaling, considered in this work, at the energies of ion beams at the LHC. For a fixed target with a beam energy of $2.76 \mathrm{TeV}$ per nucleon and $\mathrm{Z}=3$ from formula (4) we obtain $X=1.75$ for production angle 28 degrees.

\section{Conclusion}

The extrapolation of the scaling observed in the parton model for subthreshold antiproton production in the collision of heavy ions to high energies was performed, in order to obtain estimates of the cross section and yield for the production of particles with masses up to $16 \mathrm{TeV}$. It is proposed to investigate subthreshold phenomena in an experiment with a fixed target at the LHC.

\section{Acknowledgement}

This research was supported by the RFBR/CNRS grant 18-52-15007. 
There is no potential conflict of interest in this article

\section{References}

1. Rubakov, V. A. Multidimensional models of particle physics. Physics-Uspekhi. 46, 211-218 (2003)

2. Baldin, A. A. et al. Antiproton yield in the collision of carbon nuclei with copper nuclei at energy of 3.65 GeV/nucleon. JETP Lett. 48, 137-140 (1988)

3. Baldin, A. A. et al. Subthreshold anti-proton production in nucleon nucleus and nucleusnucleus collisions. Nucl. Phys. A. 519, 407-411 (1990)

4. Carroll, J. B. et al. Subthreshold antiproton production in $28 \mathrm{Si}$ collisions at $2.1 \mathrm{GeV} /$ nucleon. Phys. Rev. Lett. 62, 1829-1832 (1989)

5. Chiba, J. et al. Enhancement of subthreshold antiproton productions in deuteron induced reactions. Nucl. Phys. A. 553, 771-774 (1993)

6. Schroeter, A. et al. Subthreshold antiproton production in heavy ion collisions at SISenergies Nucl. Phys. A. 553, 775-778 (1993)

7. Shor, A., Perez-Mendez, V., Ganezer, K. Internal nuclear momentum and subthreshold antiproton production in p-nucleus and nucleus-nucleus collisions. Nucl.Phys. A. 514, 717-733 (1990)

8. Kurepin, A. B., Shileev, K. A., Topilskaya, N. S. Collective parton correlations in kaon and antiproton production by relativistic nuclei collisions. Genshikaku Kenkyu, Tokyo, 41, 177$182(1997)$ 
9. Baldin, A. A. et al. Mass dependence of antiproton production in nucleus-nucleus collisions at $3.65 \mathrm{GeV} /$ nucleon. Nuovo Cimento A. 108, 139-146 (1995)

10. Kurepin, A. B., Shileev, K. A., Topilskaya, N. S. Collective effects in kaon production by relativistic nuclei collisions. Acta Physica Polonica B. 27, 3077-3080 (1996)

11. Kurepin, A. B., Topilskaya, N. S., Golubeva, M.B. Charmonium production in fixed-target experiments with SPS and LHC beams at CERN. Phys. Atom. Nucl. 74, 446-452 (2011)

12. Brodsky, S. J., Fleuret, F., Hadjidakis, C., Lansberg, J. P. Physics opportunities of a fixedtarget experiment using LHC beams. Phys. Rep. 522, 239-255 (2013)

13. Kusina, A. et al. Probing the high-x content of the nuclei in the fixed-target mode at the LHC. Preprint at http://arXiv.org/quant-ph/1901.07950 (2019). 


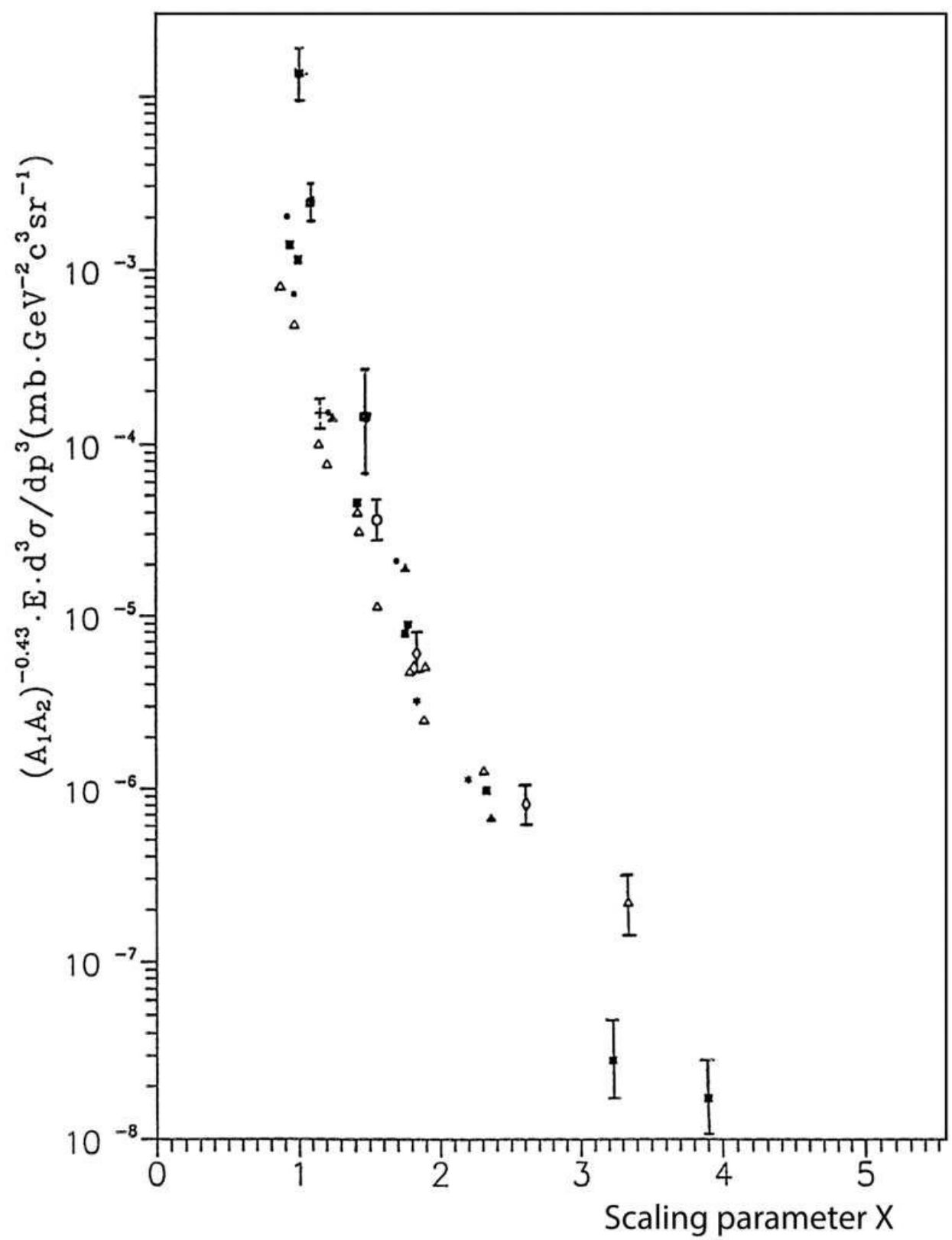

Fig. 1.

Fig. 1. Systematics of Lorentz invariant antiproton subthreshold production cross section dependence on the scaling parameter $\mathrm{X}$ with $\mathrm{Z}=1$ for incident protons, $\mathrm{Z}=1.3$ for deuterons, $\mathrm{Z}=2$ for carbon ions, $\mathrm{Z}=3$ for heavy nuclei. Points $\mathrm{p}+\mathrm{C}$, open circles $\mathrm{d}+\mathrm{C}$, crosses $\mathrm{C}+\mathrm{C}$ and $\mathrm{C}+\mathrm{Cu}^{2,3}$, triangles $\mathrm{p}+\mathrm{C}$ and $\mathrm{d}+\mathrm{C}^{5}$, squares $\mathrm{p}+\mathrm{Cu}^{7}$, rhombs $\mathrm{Si}+\mathrm{Si}$ ${ }^{4}$, stares $\mathrm{Ne}+\mathrm{Sn}$ and $\mathrm{Ni}+\mathrm{Ni}^{6}$. Only some statistical errors are presented to show the order of uncertainty for the measurements of subthreshold production. 


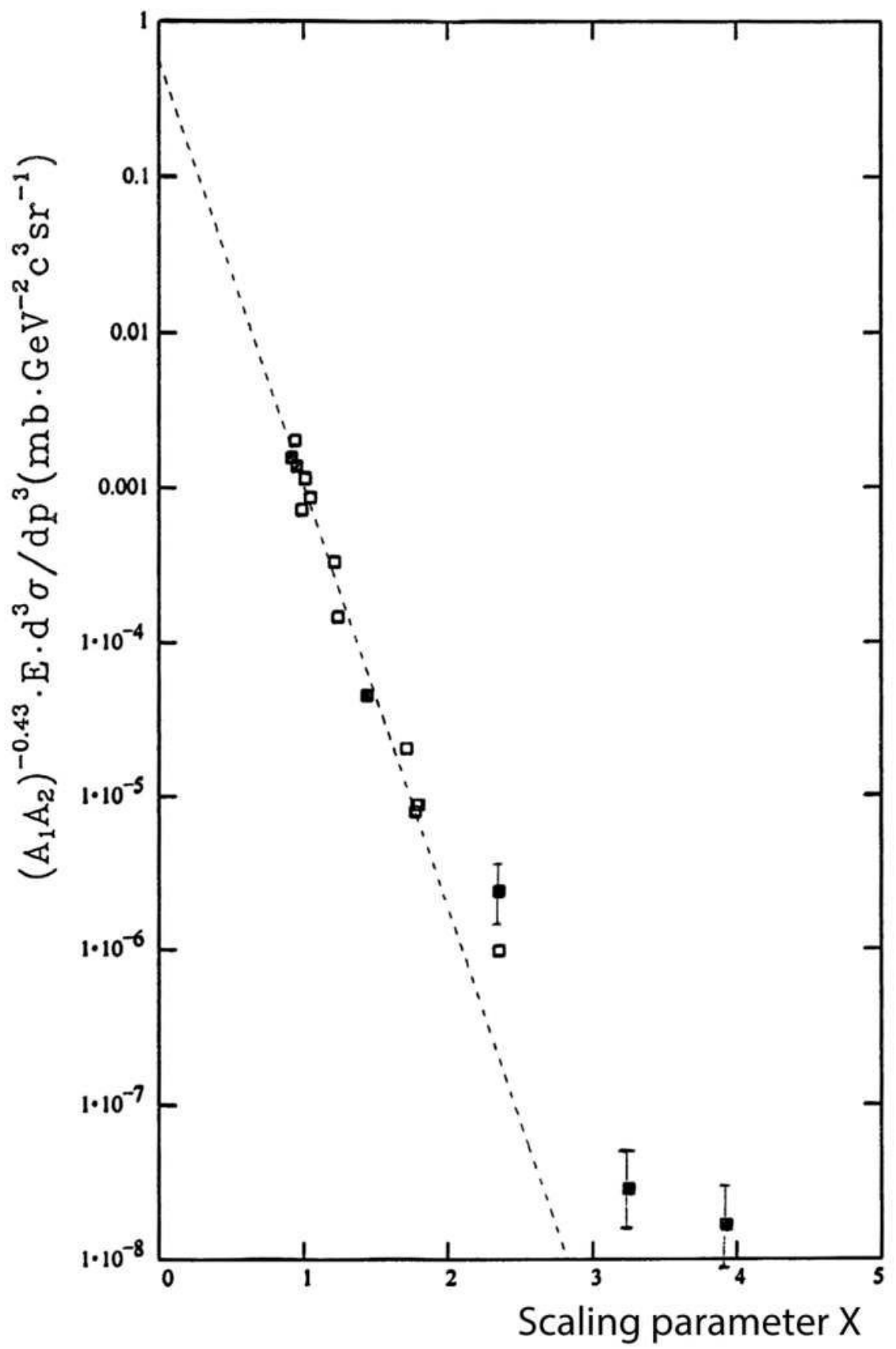

Fig. 2

Fig. 2. $\chi^{2}-$ fit to the invariant antiproton production cross section dependence on $\mathrm{X}$ scaling parameter. Only data for the interaction of protons with carbon ( open marks ) and with copper (filled marks ) are shown 


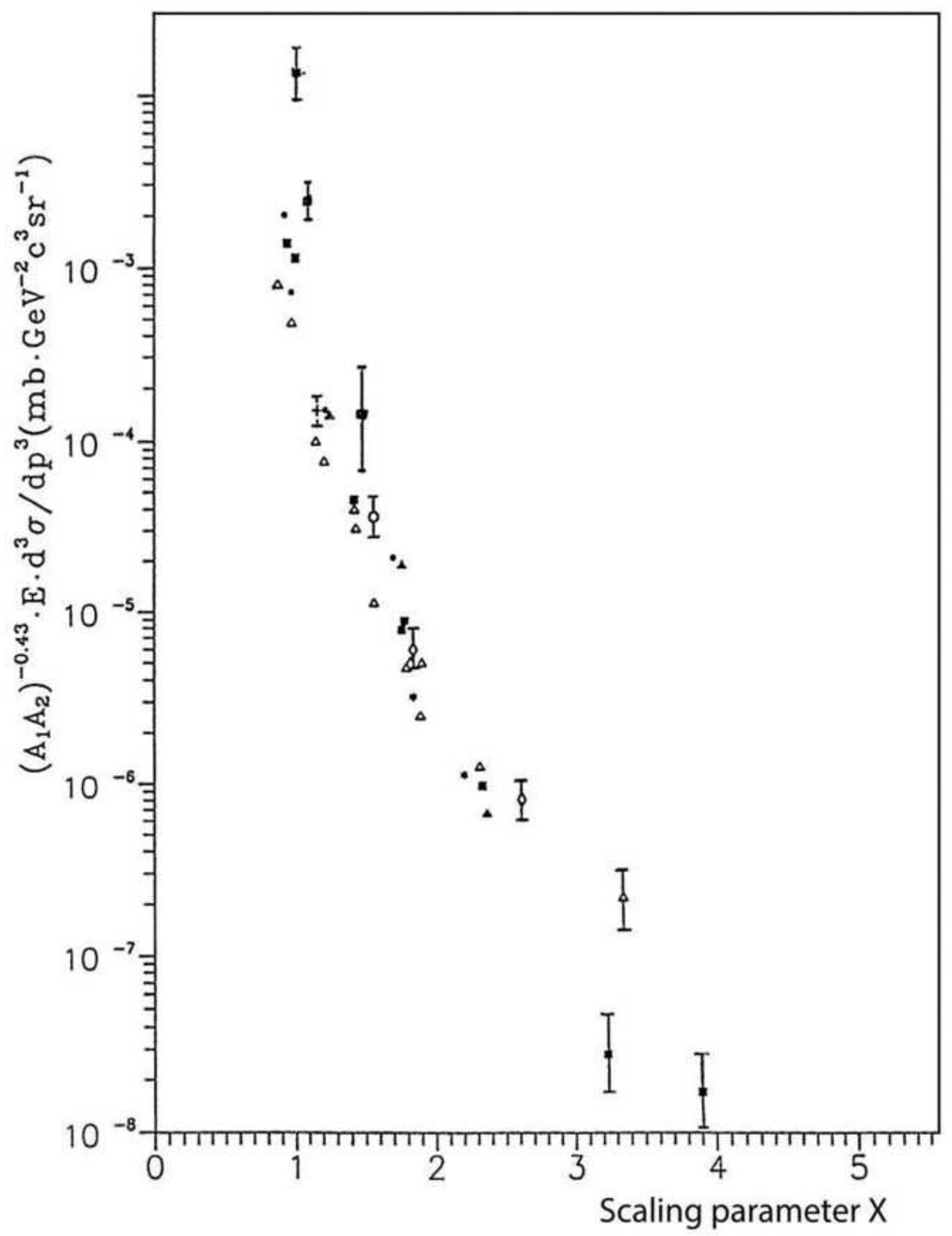

Figure 1

Systematics of Lorentz invariant antiproton subthreshold production cross section dependence on the scaling parameter $X$ with $Z=1$ for incident protons, $Z=1.3$ for deuterons, $Z=2$ for carbon ions, $Z=3$ for heavy nuclei. Points $p+C$, open circles $d+C$, crosses $C+C$ and $C+C u 2,3$, triangles $p+C$ and $d+C 5$, 
squares $\mathrm{p}+\mathrm{Cu} 7$, rhombs $\mathrm{Si}+\mathrm{Si} 4$, stares $\mathrm{Ne}+\mathrm{Sn}$ and $\mathrm{Ni}+\mathrm{Ni} 6$. Only some statistical errors are presented to show the order of uncertainty for the measurements of subthreshold production.

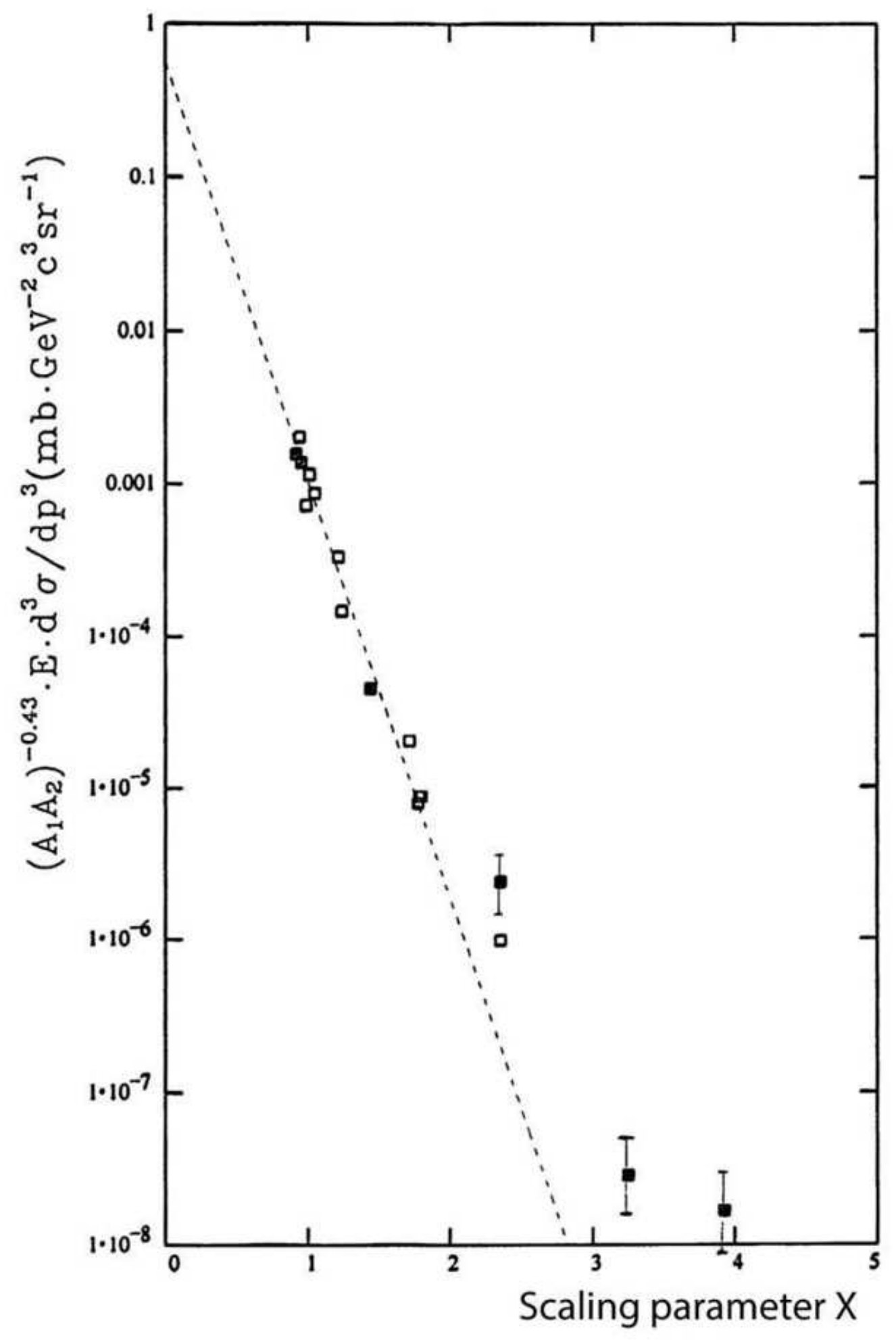

Figure 2

$\chi 2$ - fit to the invariant antiproton production cross section dependence on X-scaling parameter. Only data for the interaction of protons with carbon (open marks) and with copper (filled marks) are shown 


\section{Supplementary Files}

This is a list of supplementary files associated with this preprint. Click to download.

- KurepinSupplementaryinformation.docx 